\title{
Investigation of the effect of Delayed Auditory Feedback and transcranial Direct Current Stimulation (DAF-tDCS) treatment for the enhancement of speech fluency in adults who stutter: a Randomized Controlled Trial
}

\section{Narges Moein}

Iran University of Medical Sciences https://orcid.org/0000-0002-5102-5385

Reyhane Mohamadi ( $\nabla$ mohamadi.r@iums.ac)

Iran University of Medical Sciences

\section{Reza Rostami}

University of Tehran

\section{Michael Nitsche}

Libniz research centre for working environment and human factors

\section{Reza Zomorrodi}

Temetry Centre for Trapeutic Brain Intervention, University of Toronto

\section{Amir Ostadi}

University of Waterloo

Abbasali Keshtkar

Tehran University of Medical Sciences

\section{Research}

Keywords: Delayed Auditory Feedback, Stuttering, trans-cranial Direct Current Stimulation, speech fluency, adults who stutter

Posted Date: May 20th, 2020

DOI: https://doi.org/10.21203/rs.3.rs-29391/v1

License: (c) (i) This work is licensed under a Creative Commons Attribution 4.0 International License.

Read Full License 


\section{Abstract}

Background: With a population prevalence of one percent, stuttering is among the main speech pathology-related topics of research. Adults who stutter may benefit from transcranial direct current stimulation (tDCS) as an adjunctive intervention for enhancing speech fluency. In this study, Delayed Auditory Feedback (DAF) was combined with tDCS applied over the superior temporal gyrus. It was anticipated that intervention caused improvements of speech fluency become more stable.

Methods: A randomized, double-blind, sham-controlled clinical trial was conducted to investigate the effectiveness of intervention in enhancing speech fluency. Fifty participants were randomly allocated the intervention or control group. In the intervention group, participants received DAF combined with anodal tDCS, while the control group was exposed to sham tDCS simultaneously with DAF. Each subject participated in six intervention sessions. Speech fluency was assessed before intervention as baseline, as well as immediately, one week and six weeks after intervention.

Results: In the intervention group, the percentage of stuttered syllables was significantly reduced immediately, one week and six weeks after the intervention, as compared with the control group. The scores of the Stuttering Severity Instrument, also showed a significant reduction in the intervention group compared with the control group. No significant difference was found in the Overall Assessment of the Speaker's Experience of Stuttering questionnaire scores between the two patient two groups after intervention.

Conclusion: The results of this study propose anodal tDCS as an adjunctive method to increase speech fluency in stuttering for a prolonged time course after intervention, when combined with fluency therapy.

Trial registration: This trial was registered in ClinicalTrial.gov before recruiting the subjects. The registration number is NCT03990168 and the date of registration is June 18, 2019.

https://clinicaltrials.gov/ct2/show/NCT03990168

\section{Introduction}

\section{Background}

With a population prevalence of one percent, stuttering has gained critical attention and is among the main speech pathology-related topics of research in academia in the past few years [1,2]. Although the issue of stuttering has seen advances in terms of diagnosis and treatment, there still exist major limitations such as instability of treatment outcomes and lack of knowledge about therapeutic long-term results which need to be addressed [3]. As reported in the literature by some recently conducted research work, adults who stutter may benefit from non-invasive brain stimulation as an adjunctive intervention for enhancing the results of speech fluency treatment [4]. One of the available tools that has recently received considerable attention in this regard is transcranial direct current stimulation (tDCS). In this 
noninvasive brain stimulation technique, a weak constant current of 1-2 $\mathrm{mA}$ is applied through the scalp and results in alteration of cortical excitability and activity [5].

In recent years, studies have shown that tDCS enhances speech and language skills in patients with aphasia or apraxia [6-10]. Naming skills improved after applying tDCS in patients with chronic aphasia who had non-fluent speech $[6,9]$ and the performance of patients with apraxia improved with respect to oral production and language tasks $[7,8]$. In these studies, the anodal electrodes were placed over the frontal cortex and frontotemporal areas (Broca's region) [6-9]. Recent studies have shown that tDCS increases speech fluency also in adults who stutter when applied during a fluency intervention $[4,11]$. According to findings that show that the frontal cortex shows abnormalities in individuals who stutter [12], this potential target area for tDCS has been tackled in a recently conducted study [4]. Chesters, Watkins, and Mottonen investigated the effect of five sessions of anodal tDCS over the left inferior frontal gyrus during a speech fluency intervention on stuttering. They report a significant improvement of speech fluency in the treatment group who received anodal tDCS combined with the fluency intervention in comparison with a respective sham tDCS group. It was concluded that simultaneous usage of tDCS with fluency intervention can enhance speech fluency in stutterers.

One method which has been shown to be effective in enhancing speech fluency in individuals who stutter is Delayed Auditory Feedback (DAF). DAF enhances fluency via changing the speech rate by altered timing of feedback [13]. As reported in a recent study, enhancement of speech fluency in individuals who undergo DAF is associated with increased activity of the primary and secondary auditory areas in the superior temporal gyrus [13]. These areas show bilateral under-activation in stutterers, compared to those without respective symptoms, as shown by functional neuroimaging methods [12]. One main issue of DAF is that it is time-consuming, and it has restricted long-term results [14]. To overcome these limitations, and therefore, to enhance the efficacy of the treatment, one approach is to use adjunctive interventions. In this study, DAF, as a speech fluency intervention, was combined with tDCS applied over the superior temporal gyrus (electrode position T3 of the 10-20 international system). By considering the fact that with tDCS, cortical functions can undergo plasticity-like changes which can outlast the stimulation period, it was anticipated that intervention-caused improvements of speech fluency become more stable [15].

In tDCS, electrical current flows between two or more electrodes, a negatively charged cathode and a positively charged anode, which are positioned at specified locations on the scalp. Applying this current causes subtle changes in the resting membrane potential of cortical neurons in the underlying brain tissue [5]. Specifically, with standard protocols, effects under the cathode (cathodal stimulation) lead to hyperpolarization of respective neuronal compartments and decrease of neuronal excitability, while effects under the anode (anodal stimulation) result in depolarization and therefore increase of excitability, at macroscopic levels [16]. Neuroplastic effects which depend on alterations of glutamatergic, and GABAergic activity, emerge some minutes after stimulation $[17,18]$. Similar to the acute membrane polarization effects, anodal and cathodal tDCS result in excitability-enhancing and excitability-reducing plasticity, respectively $[15,19,20]$. 
Because of underactivitation in the primary and secondary auditory areas in people who stutter while speaking [12], in this study, we hypothesized that stimulation of superior temporal gyrus may be effective as an adjunctive method to fluency training via DAF to enhance speech therapy success in adults who stutter. In addition, in order to investigate the long-term outcomes, we assessed the outcomes six weeks after intervention. This hypothesis was systematically tested in the present project.

\section{Specific objectives}

The purpose of this study was to investigate the effect of adjunctive non-invasive brain stimulation on speech fluency in adult stutterers. In order to fulfill this goal, we recruited two groups of stuttering adult participants, i.e. a control group and an intervention group. In the control group, the participants received sham stimulation during DAF, whereas the participants in the intervention group received anodal tDCS simultaneously with DAF as a fluency intervention. We hypothesized that the fluency intervention combined with anodal tDCS over the superior temporal gyrus would be more effective as compared to the sham tDCS control condition in enhancing speech fluency in stutterers.

Measures of speech fluency were collected at 5 time points: one week and immediately before intervention as well as immediately, one week and six weeks after intervention. Outcome measures were compared between control and intervention groups across these time points. The primary outcome measure was the mean score of the percentage of stuttered syllables (SS \%) [21]. According to this, the primary objective of this study was to compare the mean score of the SS \% between the intervention and the control group at 5 assessment time points. Besides, we had two secondary outcomes measures; 1 . the mean score of Stuttering Severity Instrument-Fourth Edition (SSI-4) [22], 2. the mean score of OASES questionnaire [23]. Thus two secondary objectives were; 1 . Comparison of the mean score of stuttered syllables, duration, physical concomitant behaviors, and total score of the SSI-4 between the intervention and the control group at 5 assessment time points 2. Comparison of the means of the general information section, reaction to stuttering section, communication in daily situations section, quality of life section, total impact score and impact rating of the OASES questionnaire between the intervention and control groups at 5 assessment time points.

\section{Methods}

\section{Trial design}

A randomized, double-blind, sham-controlled clinical trial was conducted to investigate the effectiveness of anodal tDCS combined with DAF in enhancing speech fluency in adults who stutter. Fifty participants were randomly allocated to one of the groups. In the intervention group, participants received DAF combined with anodal tDCS, while the control group was exposed to sham tDCS simultaneously with DAF.

\section{Participants}


The study population consisted of adults with stuttering problems. Since this study addressed adults with at least moderate levels of stuttering, a speech-language pathologist assessed the severity of stuttering in each subject to determine whether he/she could be enrolled. In order to assess stuttering severity, the SSI-4 questionnaire was used. Inclusion criteria included history of developmental stuttering, diagnosed with moderate to severe stuttering, right-handed, age 18 to 50 years (adult), native speaker of Farsi, and non-smoker. Exclusion criteria included stuttering accompanied by other speech or language disorders, having stuttering treatment within the month before intervention, hearing loss, history of neurological or psychiatric disorders, history of seizures, intake of any medication that affects brain functions, such as anti-depressants, pregnancy, breast-feeding, cranial bone defects, cranial/brain metal implants, and skin lesions. The CONSORT flow diagram for this study is shown in Fig. 1.

\section{Interventions}

In this study, two pre-intervention assessments were performed for baseline measures. These were conducted one week and immediately before intervention. Once the assessment conducted immediately before intervention was finalized, the first intervention session began. Each subject participated in six intervention sessions. After the last intervention session, immediately post-intervention assessments were carried out and one week later, the second post-intervention assessments were conducted. Afterwards, to investigate the maintenance of the treatment gains, post-intervention assessments were done six weeks after treatment termination. This single-center trial was conducted at the Vahdat Neurorehabilitation Clinic in Tehran, Iran.

Transcranial Current Direct Stimulation

Participants underwent anodal or sham tDCS according to the group they were randomly assigned to. An EEG cap was used to identify the site of stimulation. The anode electrode was positioned over the left superior temporal gyrus (T3 according to the 10-20 international system [24]), and the cathode electrode was placed over the right frontopolar region (Fp2 according to 10-20 system [24]). The electrode positions were fixed by elastic rubber straps. In the anodal group, stimulation was done by passing $1 \mathrm{~mA}$ current between two $5 \mathrm{~cm} \times 7 \mathrm{~cm}$ electrodes for a duration of 20 minutes with ramp up and ramp down intervals of 15 seconds. This setup was shown to be efficient for enhancing speech fluency in other tDCS studies $[4,25]$. A neuroConn DC-STIMULATOR was used to deliver tDCS. We used conductive rubber electrodes encased in a sponge pocket for tDCS. Physiological saline solution was used as an electrolytebased contact medium. Before placing the electrodes on the scalp, the clinician checked the skin for any skin damage or lesion. For both, anodal and sham modes, the same stimulation intensity parameters were used. However, in the sham group, the duration of stimulation was 30 seconds and the current turned off automatically after 30 seconds.

Delayed Auditory Feedback

In all six intervention sessions, a speech and language pathologist conducted a fluency intervention during stimulation. In this study, DAF was used as a fluency intervention. In order to deliver DAF, Audapter, 
which is a software package for manipulating the acoustic parameters of speech in real-time, was used [26]. The subjects performed three tasks which included oral reading, monologue, and conversation, under 60 -ms delayed auditory feedback. This 60 -ms delay has been shown to be efficient to enhance speech fluency in stuttering [27].

Safety and side effects of tDCS were assessed after each intervention session through filling in a 5 point Likert scale ( $1=$ very mild, $5=$ very severe $)$ questionnaire by the participants. The respective questions included potential side effects of tDCS, namely itching, burning, tingling, headache, fatigue, sudden mood change, difficulties to concentrate, change of visual perception, unpleasant somatosensory sensations, unpleasant visual sensations, nausea, drowsiness, feeling that stimulation persisted after the end of intervention, and one open question for any other adverse effects. Furthermore, participants were asked to guess if they received anodal or sham stimulation before any intervention and after the first intervention, as well as after the last interventions, to ensure successful blinding.

\section{Outcome measures}

In this study, the change in percentage of Stuttered Syllables (SS \%) served as primary outcome parameter. We obtained 2 baseline measurements, one week, and immediately before intervention, to guarantee symptom stability. At these two pre-intervention time points, the voice of the participants was recorded when performing three different tasks, oral reading, monologue, and conversation. Based on these data, we calculated the primary outcome measure (SS \%), which was the average of percentages in those three tasks. A ZOOM H5 handy recorder was used to record the voice of the participants. The same measurement was taken immediately, one week, and six weeks after the last intervention session (postintervention assessments). In order to prevent any potential adaptation effect, different reading materials and novel monologue and conversation topics were used in each assessment and intervention session.

Secondary outcomes were the score of Stuttering Severity Instrument-4 (SSI-4), as well as the score of the Overall Assessment of Speaker's Experience of Stuttering (OASES). In the two pre-intervention assessments, the principal investigator observed physical behavior (such as distracting sounds, facial grimaces, head movements, and arm and leg movements) which was required to calculate the score of the SSI-4. In addition, participants filled out the OASES questionnaire which is a self-report assessment tool, as the secondary outcome measure. After the second baseline assessments, the intervention was started, and was carried out over six consecutive days.

\section{Randomization}

Participants were randomly allocated to intervention and control groups. The random assignment of the participants prevented selection bias and dysbalanced confounding factors between the study arms. A computer-generated randomization method was used via the website www.randomization.com. We determined 50 subjects and four blocks of equal size to generate a random list. Each participant was given a unique ID and was assigned to one of the experimental groups. The person responsible for generating the random list was not involved in any other part of the trial. 


\section{Blinding}

This study was double-blind. Neither the participants nor the investigators knew which group (anodal or sham) each participant was assigned to. A sealed opaque envelopes method was used for the purpose of concealment. A clinician was given randomly generated treatment allocations within sealed opaque envelopes. Once a participant consented to enter the trial, an envelope was opened and the participant was then allocated to one of the intervention groups. Researchers who were responsible for outcome assessment and data analysis were also masked to the group assignment.

For exploring success of blinding, before any intervention and after the first intervention as well as after the last session, participants were asked which type of treatment they thought to receive, anodal tDCS or sham.

\section{Statistical methods}

All statistical analyses were performed using SPSS Statistics V22.0 and Stata 15. A sample size of 25 participants per group (50 participants in total) was estimated based on the previous study by Chester and colleagues [4], and using G*Power software [28]. This sample size was required to detect a significant difference for the relevant interaction term time $\times$ group in the primary statistical test, a 2-way mixed model ANOVA with the within-subject factors time, the between-subject factor group (anodal/sham tDCS), and the dependent variable stuttered syllables (SS \%), $a$ and $\beta$ errors of 0.05 and 0.20 respectively, and an effect size of $f=0.17$.

Two experienced raters independently counted the SS\% of all speech samples. Cohen's kappa coefficient was used to measure inter-rater reliability.

Data were analyzed by intention-to-treat (ITT) and modified intention-to-treat (mITT) approaches. In order to test the assumption that data distribution was normal, Kolmogorov-Smirnov (K-S) and Shapiro-Wilk tests were performed.

Demographic as well as baseline characteristics, and primary and secondary outcomes were assessed by descriptive statistics including measures of mean, standard deviation, median, minimum, and maximum for quantitative variables, frequency and percentage for qualitative variables. The characteristics of the participants and demographic variables in the two groups were compared at baseline using Student's ttests in case of quantitative, and the chi-square test for qualitative variables.

The effect of anodal tDCS on SS \% (the primary outcome) was assessed by a 2-way mixed-model ANOVA with stuttered syllables as dependent variable, the between-subject factor tDCS (anodal vs. sham), and the within-subject factor time (before, immediately, one week after, and six weeks after intervention). In case of significant results of the ANOVA, exploratory post-hoc Student's t-tests were performed to identify significant differences for each time point between the two groups. For the secondary outcome measurements (SSI-4 and OASES scores), the same procedures were conducted. 
Adverse effects questionnaire data were analyzed using a 2-way mixed-model ANOVA. A chi-square test was performed to assess successful blinding.

\section{Results}

Fifty people (Mean $=26.92$ year, $S D=6.23$ ) with moderate to severe stuttering met the inclusion criteria and were enrolled in the study. No drop-out occurred and all participants completed all intervention and post-assessment sessions. In order to determine inter-rater reliability, two experienced raters independently counted the SS\% of all speech samples and Cohen's kappa was calculated. The strong intraclass correlation coefficient (ICC) for the inter-rater agreement $(I C C=0.95, p<0.05)$ indicate high rating reliability.

Demographic and baseline characteristics of the participants are reported in Table 1. Group differences of quantitative demographic variables were explored by Student's t-tests (for independent samples). The results showed no significant differences between the respective variables at baseline between the two groups ( $p>0.05$ for all variables). Additionally, the standardized mean difference (SMD) was calculated to investigate the mean differences of primary and secondary outcomes between the two groups.

Comparison of the primary outcome measure SS\% for the baselines and immediately, one week, and six weeks after the treatment resulted in a significant main effect of group $\left(F(1,49)=65.95, p=0.001, \eta_{p}^{2}\right.$ $=0.85 ;$ Fig. 1). The results also showed that the interaction between time and group was significant ( $F(1$, $\left.48)=74.41, p=0.001, \eta_{p}^{2}=0.77\right)$. As compared to baseline, SS\% values decreased in the anodal tDCS group for the post intervention time points 3,4 , and 5 (immediately, one week and six weeks after intervention), and in the sham group for the time points 3 (immediately after intervention). For the between group comparisons, SS\% were significantly smaller in the anodal tDCS group at time points 3, 4, and 5 (Table 2).

The results of the SSI-4 (one of the secondary outcome measures) showed a significant main effect of group $\left(F(1,49)=59.62, p=0.001, \eta_{p}^{2}=0.52 ; F i g .2\right)$, and a significant time $\times$ group interaction as well ( $F(1$, $48)=123.55, p=0.001, \eta_{p}^{2}=0.72$ ). As compared to baseline, SSI-4 scores reduced in the anodal tDCS group for the post intervention time points 3,4 , and 5 (immediately, one week and six weeks after intervention), and in the sham group for the time points 3 (immediately after intervention). For the between group comparisons, SSI-4 score were significantly smaller in the anodal tDCS group at time points 3, 4, and 5. These findings are shown in Table 2. Additionally, the results of the SSI-4 subscores showed a significant main effect of group for frequency $(F(1,49)=35.85, p=0.001)$, and also a significant time $\times$ group interaction $(F(1,48)=34.85, p=0.001)$. As compared to baseline, frequency values reduced in the anodal tDCS group for the post intervention time points 3,4 , and 5 (immediately, one week and six weeks after intervention), and in the sham group for the time point 3 (Table 3). For the between group comparisons, frequency scores were significantly smaller in the anodal tDCS group at time points 3,4 , and 5. Moreover, for duration, a significant main effect of group $(F(1,49)=19.78, p=$ $0.001)$, and also a significant time $\times$ group interaction $(F(1,48)=32.49, p=0.001)$ was revealed. As 
compared to baseline, duration scores were reduced in the anodal tDCS group for the post intervention time points 3, 4, and 5 (immediately, one week and six weeks after intervention), while in the sham group no statistically significant differences were obtained for the post intervention time points (Table 3). For the between group comparisons, duration scores were significantly smaller in the anodal tDCS group at time points 4 and 5 (one week and six weeks after intervention). For physical concomitants, the results showed a significant main effect of group $(F(1,49)=25.95, p=0.001)$, and a significant time $\times$ group interaction $(F(1,48)=19.68, p=0.001)$. As compared to baseline, physical concomitants scores were reduced in the anodal tDCS group for the post intervention time points 3,4 , and 5 (immediately, one week and six weeks after intervention), while in the sham group, no statistically significant differences in post intervention time points were present (Table 3). For the between group comparisons, no significant differences were found in physical concomitants scores between the anodal tDCS and sham groups at the post intervention time points.

The other secondary outcome measure was the OASES score, which was assessed at the same five assessment points (one week and immediately before intervention at baseline, and immediately, one week and six weeks after intervention). We found no statistically significant main effect of group ( $F(1$, $49)=0.28, p=0.60, \eta_{p}^{2}=0.006$; Fig. 3), but a significant interaction between time and group $(F(1,48)=$ $\left.67.20, p=0.001, \eta_{p}^{2}=0.58\right)$. As compared to baseline, OASES scores decreased in the anodal, and sham tDCS groups for the post intervention time points 3 and 5 (immediately and six weeks after intervention). For the between group comparisons, no significant differences were found in the overall OASES scores (Table 2). The results of the OASES subscores showed a significant main effect of group in general information $(F(1,49)=103.99, p=0.001)$, and also a significant time $\times$ group interaction $(F(1,48)=58.6$, $p=0.001)$. As compared to baseline, general information scores decreased in the anodal tDCS group for the post intervention time points 3,4 , and 5 (immediately, one week and six weeks after intervention), and in the sham group for the time points 3 and 5 (Table 3 ). For the between group comparisons, general information scores were significantly smaller in the anodal tDCS group at time point 5 (six weeks after intervention). Additionally, the findings showed a significant main effect of group in reactions to stuttering $(F(1,49)=43.98, p=0.001)$, and a significant time $\times$ group interaction $(F(1,48)=107.31, p=$ 0.001). As compared to baseline, reactions to stuttering values decreased in the anodal tDCS group for the post intervention time points 3 and 5 (immediately and six weeks after intervention), while in the sham group, there were no statistically significant differences between baseline, and post intervention time points (Table 3 ). For the between group comparisons, no significant differences were found in reactions to stuttering scores between the anodal tDCS and sham groups at post intervention time points. Moreover, there was a statistically significant main effect of group in communication in daily situations $(F(1,49)=43.10, p=0.001)$, and also a significant time $\times$ group interaction $(F(1,48)=76.04, p=0.001)$. As compared to baseline, communication in daily situations values decreased in the anodal tDCS group for the post intervention time points 3 and 5 (immediately and six weeks after intervention), while for the sham group, no statistically significant differences of post intervention time points were obtained (Table 3). For the between group comparisons, no significant differences were found in communication in daily situations values between the anodal tDCS and sham groups at post intervention time points. 
Finally, the results showed a significant main effect of group in quality of life $(F(1,49)=38.51, p=0.001)$, and also a significant time $\times$ group interaction $(F(1,48)=72.35, p=0.001)$. As compared to baseline, quality of life scores decreased (in this scale, lower values are indicating higher quality of life) in the anodal, but not sham tDCS group for the post intervention time points 3 and 5 (immediately and six weeks after intervention) (Table 3). For the between group comparisons, no significant differences were found in quality of life scores between the anodal tDCS and sham groups at post intervention time points.

The results of the adverse effects questionnaire analysis showed no significant main effects of group ( $F$ $(1,49)=2.76, p=0.07)$, or time $(F(1,5)=1.78, p=0.17)$, and no significant time $\times$ group interaction as well $(F(1,48)=6.46, p=0.06)$. Adverse effects were limited to mild and very mild symptoms such as itching and burning sensation at the application sites.

In order to investigate successful blinding, a chi-square test was applied. The findings indicated no significant difference between the two groups with respect to guessing the intervention condition $\left(\chi^{2}(1\right.$, $\mathrm{N}=50)=0.08 ; p=0.77$ ).

\section{Discussion}

We conducted a randomized controlled trial with follow-up measures to explore the effectiveness of tDCS combined with DAF to improve speech fluency in adults who stutter. The impact of the combined treatment on symptoms was evaluated by SS\% as primary outcome parameter. Maintenance of the treatment outcomes was examined for up to six weeks to exclude only short-lasting effects with limited clinical value [2]. The results showed that SS\% was significantly reduced at the three post-intervention assessments in the anodal tDCS group as compared to the sham group. Additionally, the effect size (Cohen's $d>0.8$ ) shows a large effect of this intervention.

In addition, we obtained the SSI-4 score before and after treatment. This score includes additional information, such as changes in the duration of stutter moments and physical concomitants of stuttering. These parameters disrupt speech fluency and have negative effects on communication. Reduction of respective behaviors, as expected, are relevant for the enhancement of communication effectiveness in adults who stutter [22]. The SSI-4 score was significantly decreased at the three postintervention assessments in the anodal group, as compared to the sham group. These findings are consistent with those of the primary outcome measure. The reduction in two subscores of the SSI-4 (frequency and duration) at the post-intervention assessments in the anodal group was larger than that in the sham group, showing positive effects of tDCS on these parameters. In contrast, the reduction of the physical concomitants subcsore was relatively minor, and present for the anodal tDCS group versus baseline, but not with respect to the between group comparisons. One reason for this minor effect might be that the six-week interval covered by the outcome measures was not sufficiently long for this effect to emerge. 
Finally, we surveyed the impact of the clinical effect of the intervention on the psycho-social impact of living with stuttering by the OASES questionnaire. This questionnaire measures the effect of stuttering on four different aspects, namely general information, reactions to stuttering, communication in daily situations, and quality of life [23]. The results show that the total OASES score was significantly reduced at the immediately after intervention and follow-up assessments as compared to baseline in the two study groups. The between group comparisons conducted for respective subscores showed that this reduction was mainly caused by the general information subscore of the OASES questionnaire, and may be due to additional information about possible causes of stuttering, factors affecting stuttering, and potential treatment methods delivered to the participants during the course of the study.

The results of this study show that anodal tDCS can increase speech fluency for a prolonged time course after intervention, when combined with fluency therapy. The direction of these results is consistent with previous studies in stuttering [4], but also tDCS studies in which the impact of stimulation on other speech-related symptoms was explored $[10,29,30]$. In summary, our findings propose anodal tDCS as an adjunctive method to increase speech fluency in stuttering, particularly in adults whose behavioral treatments have failed.

In this study, a combination of behavioral intervention and tDCS was used. The effect of tDCS alone could be investigated in future studies. Adding functional neuroimaging methods it future studies would be valuable to provide mechanistic information about the mode of action of the intervention. Additionally, further studies are needed to investigate whether an extension of interventions would result in stronger effects (better improvement of fluency) and/or longer lasting positive outcomes of the treatment.

\section{Conclusions}

The results of this study showed an adjunctive effect of tDCS, when combined with DAF, on stuttering. The combined treatment led to a significant reduction of the percentage of stuttered syllables and duration of stuttering moments and these results were relatively long-lasting after intervention.

\section{Abbreviations}

tDCS: transcranial Direct Current Stimulation; DAF: Delayed Auditory Feedback; SS \%: percentage of Stuttered Syllables; SSI-4: Stuttering Severity Instrument-Forth Edition; OASES: Overall Assessment of the Speaker's Experience of Stuttering

\section{Declarations}

Registration

This trial was registered in ClinicalTrial.gov before recruiting the subjects. The registration number is NCT03990168 and the date of registration is June 18, 2019. 


\section{Protocol}

The protocol article of this study has been accepted in the JMIR research and protocols journal [31].

\section{Funding}

Iran University of Medical Sciences funded this work.

\section{Ethics approval and consent to participate}

This study was carried out in accordance to the ethical principles and the national norms and standards for conducting Medical Research in Iran and has the approval ID of IR.IUMS.REC.1398.352 of the Iran National Committee for Ethics in Biomedical Research.

\section{Consent for publication}

Although the investigators are free to use the study findings for educational and scientific purposes, prior to submission of the manuscript, written consent was obtained from the study sponsor. Any information about subjects is kept confidential and not available for public access. In addition, any document, data, voice recording, and other records are coded by a participant identification number, and the subject names are kept confidential.

\section{Availability of data and materials}

The datasets used and/or analyzed during the current study are available from the corresponding author on reasonable request.

\section{Competing interests}

MN is at the scientific advisory boards of Neuroelectrics, and NeuroDevice. The other authors declare that they have no competing interest. This study has received assistance from Iran National Science Foundation and has undergone peer-review by the funding body.

\section{Authors' contributions}

$\mathrm{RM}, \mathrm{RR}$, and $\mathrm{MN}$ contributed to the conception and design of the study. AK and RZ contributed in analyzing and interpretation of the data. AO wrote the MATLAB code. NM was a major contributor in 
writing the manuscript and collecting the data. MN substantively revised the manuscript. All authors read and approved the final manuscript.

\section{Acknowledgements}

We would like to thank the Iran National Science Foundation for supporting this trial.

\section{References}

1. Bloodstein O, Ratner NB. A handbook on stuttering Clifton Park, NY: Thomson Delmar Learning; 2008. A Handbook on Stuttering.

2. Guitar B, Stuttering: An integrated approach to its nature and treatment. 2013: Lippincott Williams \& Wilkins.

3. Ward D, Stuttering and cluttering: frameworks for understanding and treatment. 2017: Psychology Press.

4. Chesters J, Möttönen R, Watkins KE. Transcranial direct current stimulation over left inferior frontal cortex improves speech fluency in adults who stutter. Brain. 2018;141(4):1161-71.

5. Thair $\mathrm{H}$, et al. Transcranial direct current stimulation (tDCS): a beginner's guide for design and implementation. Front NeuroSci. 2017;11:641.

6. Baker JM, Rorden C, Fridriksson J. Using transcranial direct-current stimulation to treat stroke patients with aphasia. Stroke. 2010;41(6):1229-36.

7. Marangolo $P$, et al. Bihemispheric stimulation over left and right inferior frontal region enhances recovery from apraxia of speech in chronic aphasia. Eur J Neurosci. 2013;38(9):3370-7.

8. Marangolo $P$, et al. Electrical stimulation over the left inferior frontal gyrus (IFG) determines long-term effects in the recovery of speech apraxia in three chronic aphasics. Behav Brain Res. 2011;225(2):498-504.

9. Monti A, et al. Improved naming after transcranial direct current stimulation in aphasia. Journal of Neurology Neurosurgery Psychiatry. 2008;79(4):451-3.

10. Monti A, et al., Transcranial direct current stimulation (tDCS) and language. J Neurol Neurosurg Psychiatry, 2012: p. jnnp-2012-302825.

11. Chesters J, Enhancing speech fluency using transcranial direct current stimulation. 2016, University of Oxford.

12. Brown S, et al. Stuttered and fluent speech production: An ALE meta-analysis of functional neuroimaging studies. Hum Brain Mapp. 2005;25(1):105-17.

13. Takaso $\mathrm{H}$, et al. The effect of delayed auditory feedback on activity in the temporal lobe while speaking: a positron emission tomography study. Journal of Speech Language Hearing Research. 2010;53(2):226-36. 
14. Van Borsel J, Reunes G, Van den Bergh N. Delayed auditory feedback in the treatment of stuttering: clients as consumers. International Journal of Language Communication Disorders. 2003;38(2):119-29.

15. Nitsche MA, Paulus W. Sustained excitability elevations induced by transcranial DC motor cortex stimulation in humans. Neurology. 2001;57(10):1899-901.

16. Nitsche MA, et al. Facilitation of implicit motor learning by weak transcranial direct current stimulation of the primary motor cortex in the human. J Cogn Neurosci. 2003;15(4):619-26.

17. Nitsche $\mathrm{M}$, et al. Pharmacological modulation of cortical excitability shifts induced by transcranial direct current stimulation in humans. J Physiol. 2003;553(1):293-301.

18. Stagg CJ, et al. Polarity-sensitive modulation of cortical neurotransmitters by transcranial stimulation. J Neurosci. 2009;29(16):5202-6.

19. Nitsche MA, et al. Level of action of cathodal DC polarisation induced inhibition of the human motor cortex. Clin Neurophysiol. 2003;114(4):600-4.

20. Nitsche MA, Paulus W. Excitability changes induced in the human motor cortex by weak transcranial direct current stimulation. J Physiol. 2000;527(3):633-9.

21. Cordes AK, Ingham RJ. The reliability of observational data: II. Issues in the identification and measurement of stuttering events. Journal of Speech Language Hearing Research. 1994;37(2):27994.

22. Riley G. SSI-4 stuttering severity instrument fourth edition. Austin: Pro-Ed; 2009.

23. Yaruss JS, Quesal RW. Overall Assessment of the Speaker's Experience of Stuttering (OASES): Documenting multiple outcomes in stuttering treatment. J Fluen Disord. 2006;31(2):90-115.

24. Jasper H. Report of the committee on methods of clinical examination in electroencephalography. Electroencephalogr Clin Neurophysiol. 1958;10:370-5.

25. Chesters J, Watkins KE, Möttönen R. Investigating the feasibility of using transcranial direct current stimulation to enhance fluency in people who stutter. Brain Lang. 2017;164:68-76.

26. Cai S, et al., Tensorflow debugger: Debugging dataflow graphs for machine learning. 2016.

27. Stuart A, et al. Investigations of the impact of altered auditory feedback in-the-ear devices on the speech of people who stutter: initial fitting and 4-month follow-up. International Journal of Language Communication Disorders. 2004;39(1):93-113.

28. Faul $F$, et al. Statistical power analyses using $G^{*}$ Power 3.1: Tests for correlation and regression analyses. Behavior research methods. 2009;41(4):1149-60.

29. Holland R, Crinion J. Can tDCS enhance treatment of aphasia after stroke? Aphasiology. 2012;26(9):1169-91.

30. Sandars M, Cloutman L, Woollams AM, Taking sides: an integrative review of the impact of laterality and polarity on efficacy of therapeutic transcranial direct current stimulation for anomia in chronic poststroke aphasia. Neural plasticity, 2016. 2016. 


\section{Moein N, et al. Delayed Auditory Feedback and Transcranial Direct Current Stimulation Treatment for the Enhancement of Speech Fluency in Adults Who Stutter: Protocol for a Randomized Controlled Trial. JMIR Research Protocols. 2020;9(4):e16646.}

\section{Tables}

Table1 Demographic and baseline characteristics

\begin{tabular}{lllcccc}
\hline \multicolumn{2}{l}{ Stimulation } & Mean & SD & Median & Minimum & Maximum \\
\hline Anodal & Sex (Female \%) & $12(48 \%)$ & & & & \\
$(\mathrm{n}=25)$ & Age & 26 & 6.55 & 25 & 17 & 47 \\
& SS\% & 8.38 & 2.53 & 7.66 & 4.33 & 14.33 \\
& SSI-4 & 32.84 & 4.72 & 32 & 25 & 46 \\
& OASES & 61.02 & 10.93 & 63.60 & 39.40 & 78.60 \\
\hline Sham & Sex (Female \%) & $13(52 \%)$ & & & & \\
$(\mathrm{n}=25)$ & Age & 26.50 & 5.77 & 26 & 19 & 38 \\
& SS\% & 8.45 & 3.36 & 8 & 3.67 & 14.33 \\
& SSI-4 & 32.08 & 3 & 31 & 26 & 36 \\
& OASES & 58.93 & 14.30 & 59.60 & 38.40 & 74.80 \\
\hline
\end{tabular}

Table2 Primary and Secondary outcomes distributions in pre and post-intervention assessments by trial groups (Bonferroni-adjusted)

\begin{tabular}{cccccc}
\hline variable & Time point & $\begin{array}{c}\text { Anodal group } \\
\mathrm{n}=25\end{array}$ & $\begin{array}{c}\text { Sham group } \\
\mathrm{n}=25\end{array}$ & Cohen's d (95\% CI) & P value \\
& & & & \\
\hline \multirow{2}{*}{ SS\% } & Time point 1 & $8.38(2.53)$ & $8.45(3.36)$ & $-0.02(-0.57,0.53)$ & 0.92 \\
& Time point 2 & $8.40(2.59)$ & $8.40(3.30)$ & $0.001(-0.55,0.55)$ & 0.99 \\
& Time point 3 & $4.74(1.62)$ & $6.81(2.54)$ & $-0.97(-1.55,-0.37)$ & 0.03 \\
& Time point 4 & $5.26(1.68)$ & $8.29(3.42)$ & $-1.12(-1.71,-0.52)$ & 0.005 \\
& Time point 5 & $5.36(1.67)$ & $8.45(3.35)$ & $-1.16(-1.76,-0.56)$ & 0.005 \\
\hline \multirow{2}{*}{ SSI-4 score } & Time point 1 & $32.84(4.72)$ & $32.08(3)$ & $0.19(-0.36,0.74)$ & 0.50 \\
& Time point 2 & $32.88(4.51)$ & $31.96(3.04)$ & $0.23(-0.31,0.79)$ & 0.42 \\
& Time point 3 & $27.64(4.94)$ & $30.48(2.87)$ & $-0.70(-1.27,-0.12)$ & 0.01 \\
& Time point 4 & $27.68(5.14)$ & $31.68(2.96)$ & $-0.95(-1.53,-0.36)$ & 0.001 \\
& Time point 5 & $27.96(5.02)$ & $31.92(3.08)$ & $-0.95(-1.53,-0.36)$ & 0.001 \\
\hline OASES score & Time point 1 & $61.02(10.93)$ & $58.93(14.30)$ & $0.16(-0.39,0.71)$ & 0.06 \\
& Time point 2 & $61.48(11.01)$ & $57.89(13.90)$ & $0.28(-0.27,0.84)$ & 0.07 \\
& Time point 3 & $60.22(10.95)$ & $57.01(14.06)$ & $0.25(-0.30,0.81)$ & 0.06 \\
& Time point 4 & $60.55(11)$ & $57.22(14.06)$ & $0.26(-0.29,0.81)$ & 0.06 \\
& Time point 5 & $53.91(11.57)$ & $56.75(14.10)$ & $-0.22(-0.77,0.33)$ & 0.87 \\
\hline
\end{tabular}

Time point $1=$ One week before intervention, Time point $2=$ Immediately before intervention, Time point $3=$ Immediately after intervention, Time point $4=$ One week after intervention, and Time point $5=$ Six weeks after intervention

Table3 Mean differences (MD) from baseline per group for subscores of secondary outcomes (Bonferroni-adjusted) 


\begin{tabular}{|c|c|c|c|c|c|c|}
\hline & \multicolumn{3}{|c|}{ Anodal group $(n=25)$} & \multicolumn{3}{|c|}{ Sham group $(n=25)$} \\
\hline & $\begin{array}{c}\text { MD at time } \\
\text { point } 3(95 \% \mathrm{CI})\end{array}$ & $\begin{array}{c}\text { MD at time } \\
\text { point } 4(95 \% \\
\text { CI })\end{array}$ & $\begin{array}{l}\text { MD at time } \\
\text { point } 5(95 \% \\
\text { CI })\end{array}$ & $\begin{array}{c}\text { MD at time } \\
\text { point } 3(95 \% \mathrm{CI})\end{array}$ & $\begin{array}{c}\text { MD at time } \\
\text { point } 4(95 \% \mathrm{CI})\end{array}$ & $\begin{array}{c}\text { MD at time } \\
\text { point } 5(95 \% \mathrm{CI})\end{array}$ \\
\hline Frequency & $\begin{array}{l}-2.72 \\
(-3.28,-2.15)\end{array}$ & $\begin{array}{c}-2.24 \\
(-2.80,-1.67)\end{array}$ & $\begin{array}{c}-2.12 \\
(-2.68,-1.55)\end{array}$ & $\begin{array}{c}-0.92 \\
(-1.48,-0.35)\end{array}$ & $\begin{array}{c}-0.12 \\
(-0.68,0.44)\end{array}$ & $\begin{array}{c}0.08 \\
(-0.48,0.64)\end{array}$ \\
\hline Duration & $\begin{array}{c}-1.12 \\
(-1.44,-0.79)\end{array}$ & $\begin{array}{c}-1.20 \\
(-1.52,-0.87)\end{array}$ & $\begin{array}{c}-1.20 \\
(-1.52,-0.87)\end{array}$ & $\begin{array}{c}0.08 \\
(-0.22,0.22)\end{array}$ & $\begin{array}{c}0.09 \\
(-0.32,0.32)\end{array}$ & $\begin{array}{c}0.09 \\
(-0.32,0.32)\end{array}$ \\
\hline $\begin{array}{c}\text { Physical } \\
\text { concomitants }\end{array}$ & $\begin{array}{c}-1.41 \\
(-1.91,-0.92)\end{array}$ & $\begin{array}{c}-1.75 \\
(-2.24,-1.25)\end{array}$ & $\begin{array}{c}-1.66 \\
(-2.16,-1.17)\end{array}$ & $\begin{array}{c}-0.28 \\
(-0.76,0.20)\end{array}$ & $\begin{array}{c}-0.28 \\
(-0.76,0.20)\end{array}$ & $\begin{array}{c}-0.24 \\
(-0.72,0.24)\end{array}$ \\
\hline $\begin{array}{c}\text { General } \\
\text { information }\end{array}$ & $\begin{array}{c}-0.92 \\
(-1.81,-0.02)\end{array}$ & $\begin{array}{c}-0.76 \\
(-1.65,0.13)\end{array}$ & $\begin{array}{c}-8.48 \\
(-9.37,-7.58)\end{array}$ & $\begin{array}{c}-0.56 \\
(-1.45,0.33)\end{array}$ & $\begin{array}{c}-0.52 \\
(-1.41,0.37)\end{array}$ & $\begin{array}{c}-2.84 \\
(-3.73,-1.94)\end{array}$ \\
\hline $\begin{array}{l}\text { Reactions to } \\
\text { stuttering }\end{array}$ & $\begin{array}{c}-0.65 \\
(-0.97,-0.33)\end{array}$ & $\begin{array}{c}-0.17 \\
(-0.50,0.14)\end{array}$ & $\begin{array}{c}-5.39 \\
(-5.71,-5.07)\end{array}$ & $\begin{array}{c}-0.33 \\
(-0.65,-0.01)\end{array}$ & $\begin{array}{c}-0.06 \\
(-0.38,0.25)\end{array}$ & $\begin{array}{c}-0.05 \\
(-0.37,0.26)\end{array}$ \\
\hline $\begin{array}{l}\text { Communication in } \\
\text { daily situations }\end{array}$ & $\begin{array}{c}-0.80 \\
(-1.33,-0.26)\end{array}$ & $\begin{array}{c}-0.51 \\
(-1.05,0.01)\end{array}$ & $\begin{array}{c}-6.91 \\
(-7.44,-6.37)\end{array}$ & $\begin{array}{c}-0.60 \\
(-1.14,-0.07)\end{array}$ & $\begin{array}{c}-0.38 \\
(-0.91,0.15)\end{array}$ & $\begin{array}{c}-0.57 \\
(-1.11,-0.04)\end{array}$ \\
\hline Quality of life & $\begin{array}{c}-4.73 \\
(-9.62,0.15)\end{array}$ & $\begin{array}{c}-4.38 \\
(-9.27,0.50)\end{array}$ & $\begin{array}{l}-10.20 \\
(-15.09,-5.31)\end{array}$ & $\begin{array}{c}-5.08 \\
(-9.97,-0.19)\end{array}$ & $\begin{array}{c}-4.83 \\
(-9.72,0.05)\end{array}$ & $\begin{array}{c}-4.83 \\
(-9.72,0.05)\end{array}$ \\
\hline
\end{tabular}

Time point 3= Immediately after intervention, Time point $4=$ One week after intervention, and Time point $5=$ Six weeks after intervention

Figures 


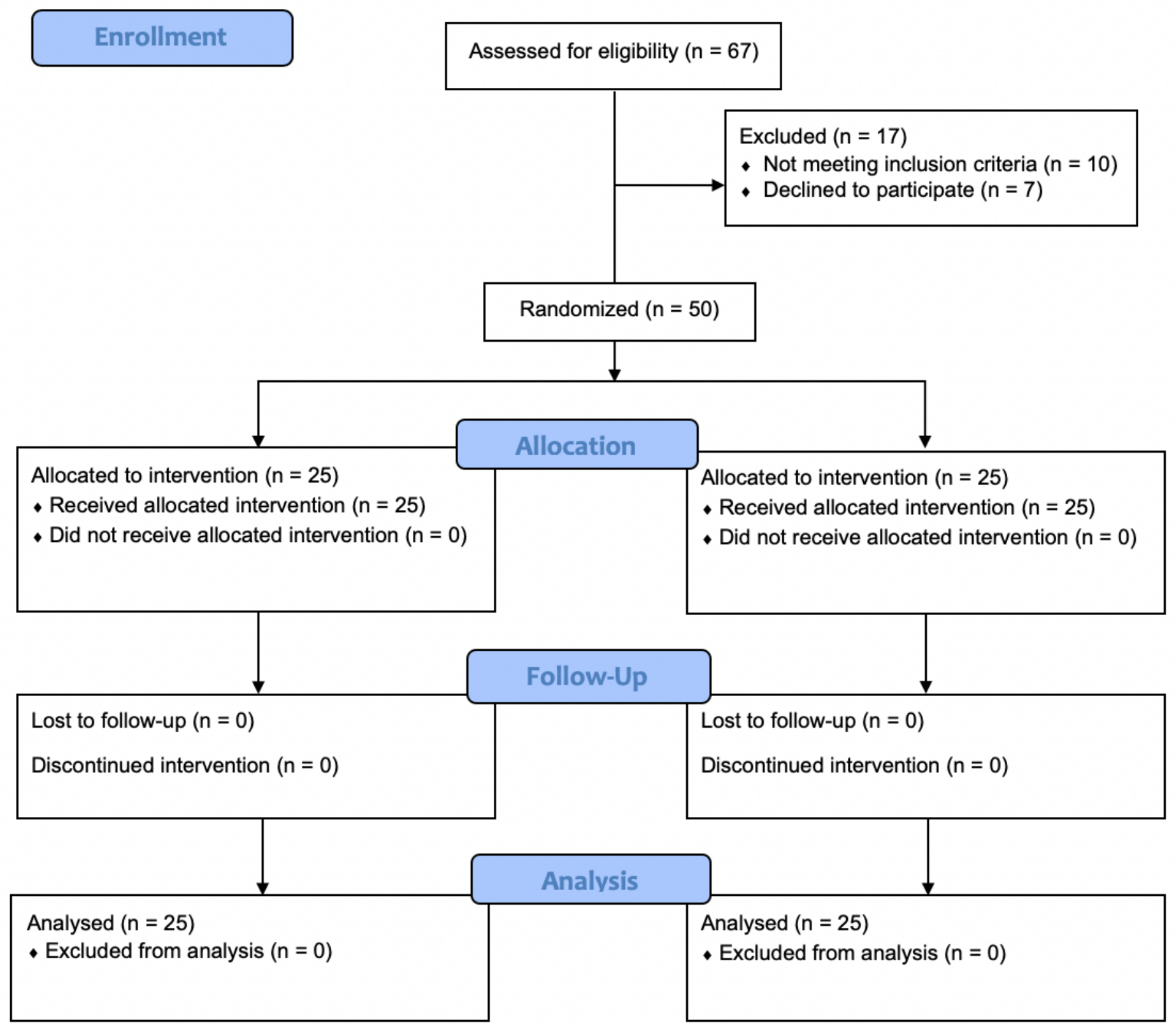

Figure 1

CONSORT flow chart of the study. The study was registered at URL:

https://clinicaltrials.gov/ct2/show/NCT03990168 (ID= NCT03990168) 


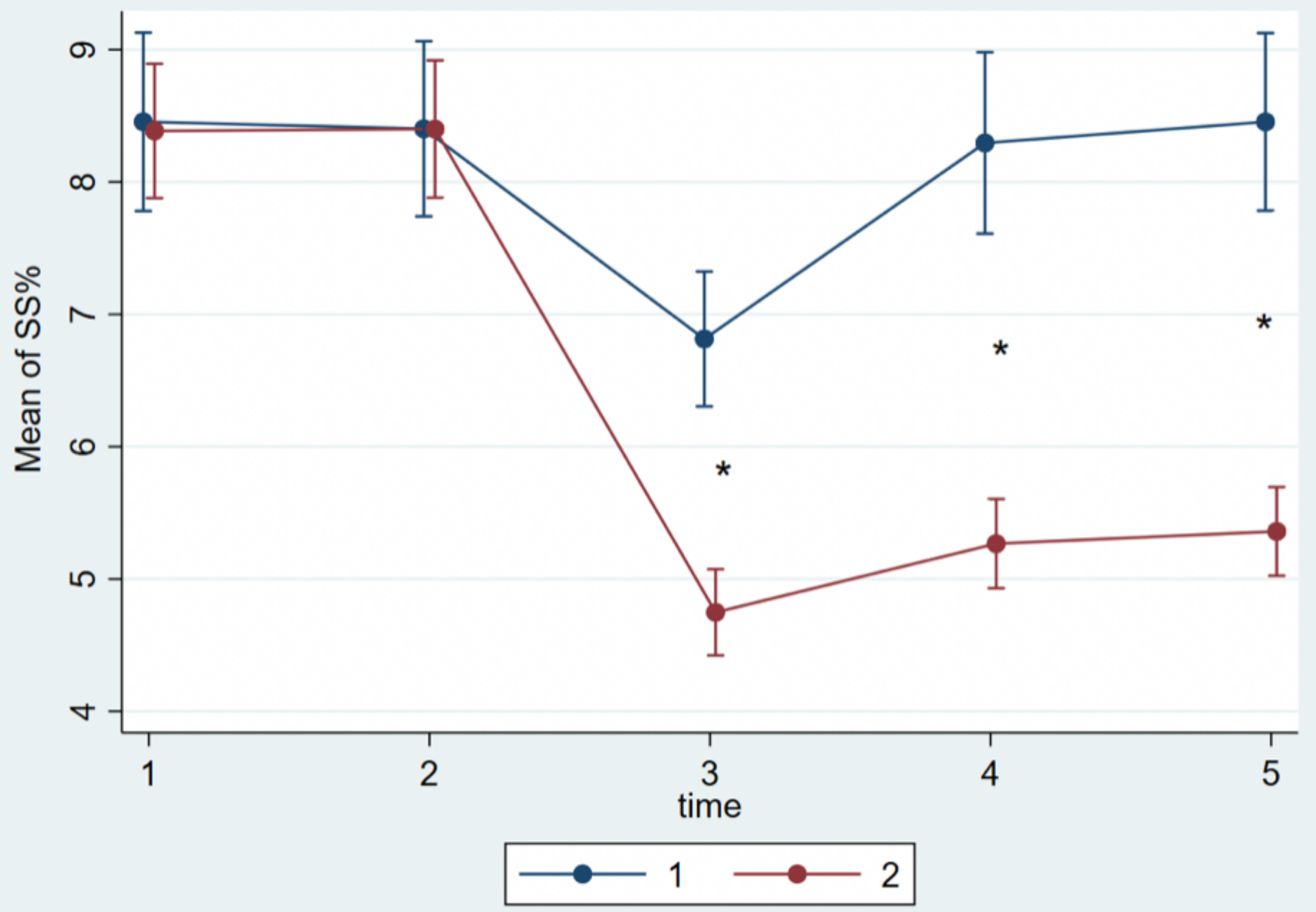

Figure 2

Effect of anodal tDCS on percentage of Stuttered Syllables (primary outcome measure). Lines show the mean of SS\% measured at two baseline points as pre-intervention assessments and 3 post-intervention points (immediately, one week, and six weeks after treatment). For SS\%, higher scores indicate larger severity. Error bars indicate standard error of the mean (SEM) (1=Sham group, 2=Anodal group). The asterisks mark significant differences between the real and sham stimulation groups. 


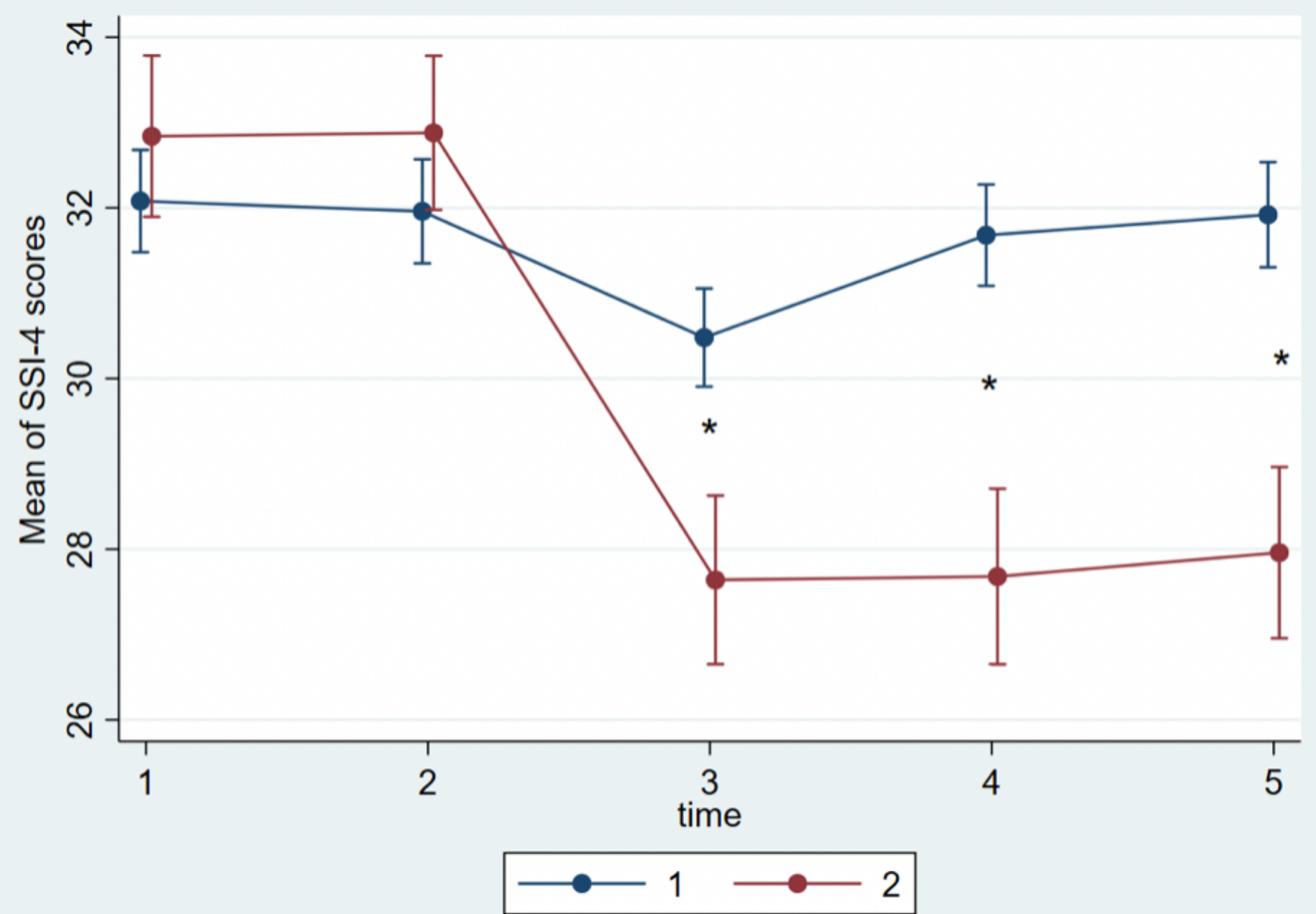

Figure 3

Effect of anodal tDCS on the SSI-4 score (secondary outcome measure). Lines show the SSI-4 scores measured at two baseline points as pre-intervention assessments and 3 post-intervention points (immediately, one week, and six weeks after treatment). The range of SSI-4 score is from 0 to 56 , higher scores indicate larger severity. Error bars indicate standard error of the mean (SEM) (1=Sham group, $2=$ Anodal group). The asterisks mark significant differences between the real and sham stimulation groups. 


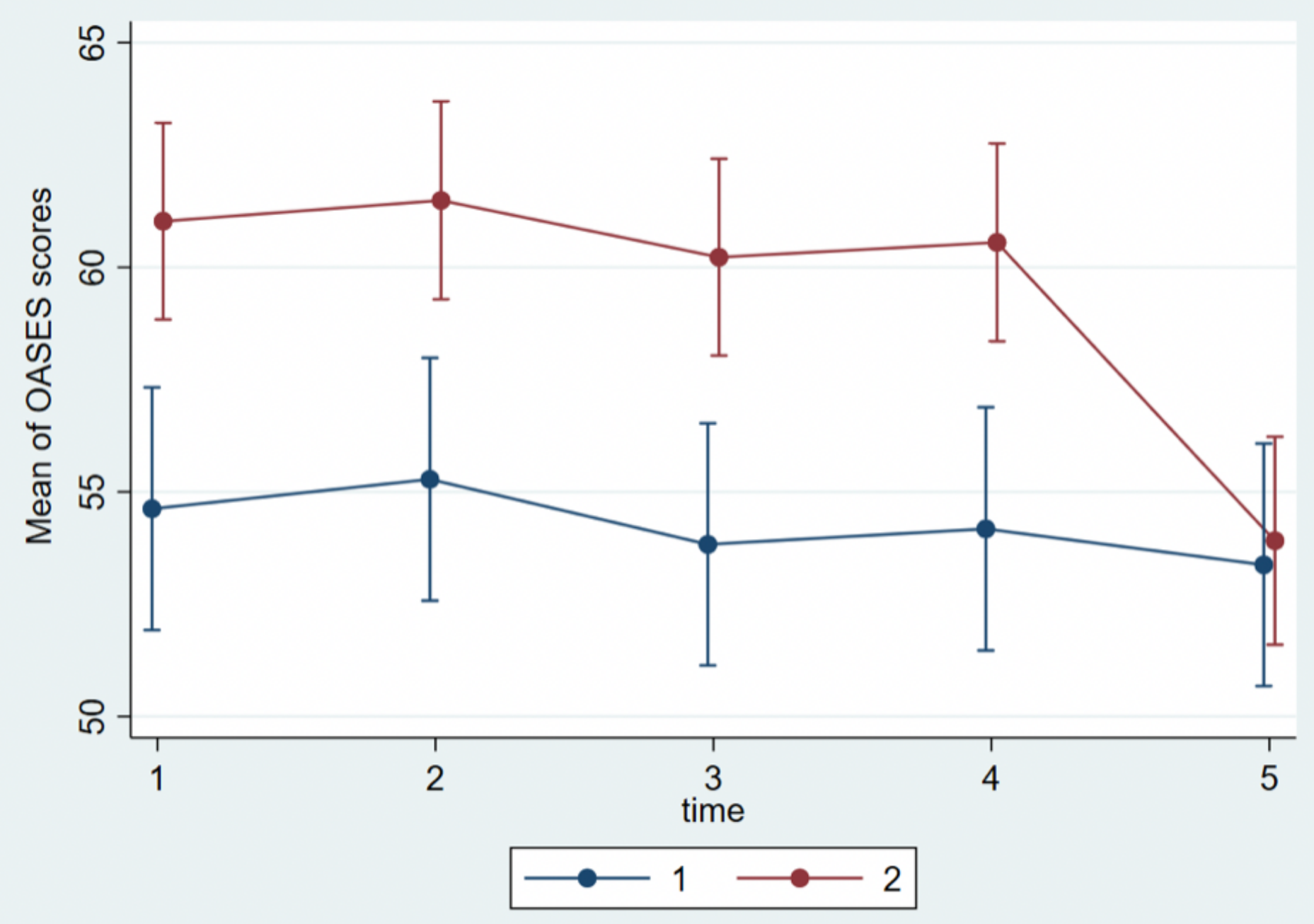

Figure 4

Effect of anodal tDCS on the OASES score (secondary outcome measure). Lines show the OASES scores measured at two baseline points as pre-intervention assessments and three post-intervention time points (immediately, one week, and six weeks after treatment). The range of OASES score is from 20 to 100, higher scores indicate more negative experiences. Error bars indicate standard error of the mean (SEM) (1=Sham group, 2=Anodal group). 